\title{
Ubiquity of collective irregular dynamics in balanced networks of spiking neurons
}

Ekkehard Ullner, Antonio Politi, and Alessandro Torcini

Citation: Chaos 28, 081106 (2018); doi: 10.1063/1.5049902

View online: https://doi.org/10.1063/1.5049902

View Table of Contents: http://aip.scitation.org/toc/cha/28/8

Published by the American Institute of Physics

\section{Articles you may be interested in}

Chaos in Kuramoto oscillator networks

Chaos: An Interdisciplinary Journal of Nonlinear Science 28, 071102 (2018); 10.1063/1.5041444

Solitary states for coupled oscillators with inertia

Chaos: An Interdisciplinary Journal of Nonlinear Science 28, 011103 (2018); 10.1063/1.5019792

Predicting tipping points of dynamical systems during a period-doubling route to chaos

Chaos: An Interdisciplinary Journal of Nonlinear Science 28, 073102 (2018); 10.1063/1.5038801

Riddling: Chimera's dilemma

Chaos: An Interdisciplinary Journal of Nonlinear Science 28, 081105 (2018); 10.1063/1.5048595

Using machine learning to replicate chaotic attractors and calculate Lyapunov exponents from data

Chaos: An Interdisciplinary Journal of Nonlinear Science 27, 121102 (2017); 10.1063/1.5010300

Overcoming network resilience to synchronization through non-fast stochastic broadcasting

Chaos: An Interdisciplinary Journal of Nonlinear Science 28, 071104 (2018); 10.1063/1.5044420

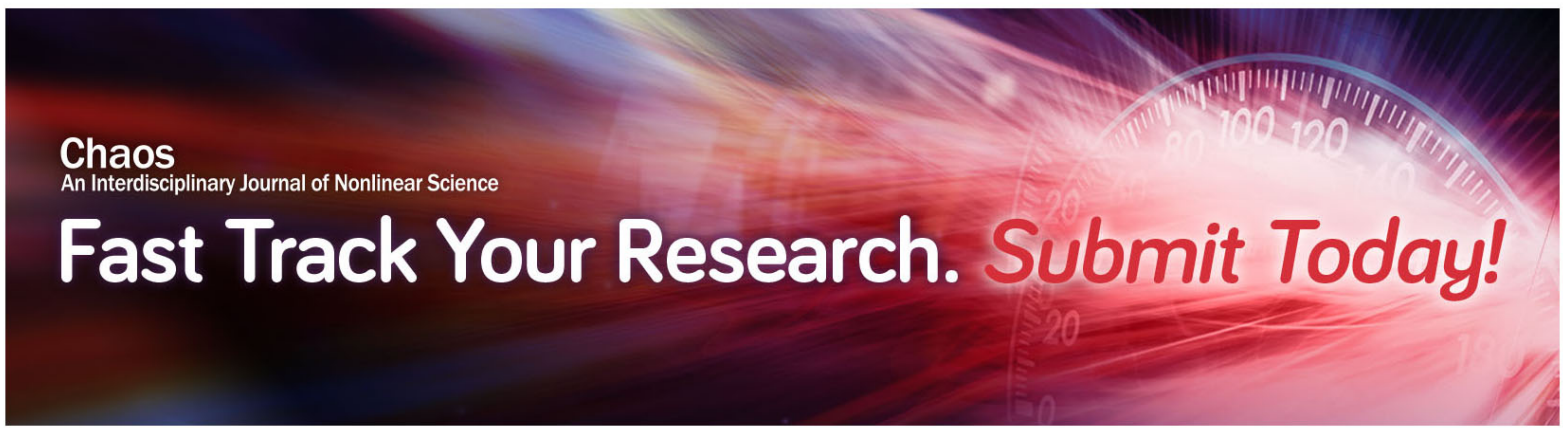




\title{
Ubiquity of collective irregular dynamics in balanced networks of spiking neurons
}

\author{
Ekkehard Ullner, ${ }^{1}$ Antonio Politi, ${ }^{1,2}$ and Alessandro Torcini ${ }^{2,3,4,5}$ \\ ${ }^{1}$ Institute for Complex Systems and Mathematical Biology and Department of Physics (SUPA), Old Aberdeen, \\ Aberdeen AB24 3UE, United Kingdom \\ ${ }^{2}$ Max Planck Institut für Physik komplexer Systeme, Nöthnitzer Str. 38, 01187 Dresden, Germany \\ ${ }^{3}$ Laboratoire de Physique Théorique et Modélisation, Université de Cergy-Pontoise, CNRS, UMR 8089, \\ 95302 Cergy-Pontoise cedex, France \\ ${ }^{4}$ Aix Marseille Univ, INSERM, INMED, and INS, Inst Neurosci Syst, 13000 Marseille, France \\ ${ }^{5}$ Istituto dei Sistemi Complessi, CNR - Consiglio Nazionale delle Ricerche, via Madonna del Piano 10, \\ I-50019 Sesto Fiorentino, Italy
}

(Received 26 July 2018; accepted 9 August 2018; published online 29 August 2018)

\begin{abstract}
We revisit the dynamics of a prototypical model of balanced activity in networks of spiking neurons. A detailed investigation of the thermodynamic limit for fixed density of connections (massive coupling) shows that, when inhibition prevails, the asymptotic regime is not asynchronous but rather characterized by a self-sustained irregular, macroscopic (collective) dynamics. So long as the connectivity is massive, this regime is found in many different setups: leaky as well as quadratic integrate-and-fire neurons; large and small coupling strength; and weak and strong external currents. Published by AIP Publishing. https://doi.org/10.1063/1.5049902
\end{abstract}

Dynamical regimes where excitation and inhibition almost balance each other are considered very important in computational neuroscience, since they are generically accompanied by strong microscopic fluctuations such as those experimentally observed in the resting state of the mammalian brain. While much is known on the balanced regime in the context of binary neurons and in networks of rate models, much less is known in the more realistic case of spiking neural networks. So far, most of the research activity on spiking neurons was restricted to diluted networks, with the goal of providing a detailed description of the underlying asynchronous regime. In this paper, we show that, contrary to the current expectations, even in the presence of a $10 \%$ dilution, the collective dynamics exhibited is characterized by a sizeable synchronization. The analysis of a suitable order parameter reveals that the macroscopic dynamics is highly irregular and remains such in the thermodynamic limit (i.e., for infinitely many neurons). The underlying form of synchronization is thereby different from the collectively regular dynamics observed in systems such as the Kuramoto model.

In spite of the many studies carried out in the last decade, a general theory of the dynamics of large ensembles of oscillators is still lacking even for relatively simple setups where the single units are assumed to be one-dimensional phase oscillators. ${ }^{1}$ A whole variety of phases has been indeed discovered which interpolate between the fully synchronous and the asynchronous regime, including chimera states, selfconsistent partial synchrony, not to speak of various clustered states. $^{2-5}$

Even though real systems are composed of a finite number of elements, we know from statistical mechanics that a meaningful identification of the different regimes can be made only in the thermodynamic limit, i.e., for an ideally infinite number of elements. In the case of dynamical systems defined on regular lattices with short range interactions, taking the limit is straightforward: it is just the matter of considering infinitely extended lattices. In networks with long-range interactions, the question is less obvious. ${ }^{6}$ Since the interaction grows with the system size, the coupling strength must be inversely proportional to the number of connections to avoid unphysical divergencies. Systems like the Kuramoto model belong to this class. ${ }^{2}$ In setups where the average coupling contribution is negligible, the coupling strength is instead assumed to scale as the inverse of the square root of the connectivity. Spin glasses are the most prominent physical systems where this latter scheme is adopted.,

The characterization of the balanced regime represents another such setup ${ }^{9}$ encountered in computational neuroscience. A theory of balanced states has been developed in ensembles of neurons characterized by a coarse-grained variable: their firing-rate. ${ }^{10-15}$ However, it is still unclear whether the resulting scenario is truly representative of what can be observed in more realistic setups.

In fact, increasing attention has been progressively devoted to simple models of excitatory and inhibitory spiking neurons, such as leaky (LIF) or quadratic (QIF) integrateand-fire neurons with the goal of mimicking the cortical activity. ${ }^{16-20}$ The most detailed theoretical analysis of spiking neurons has been proposed by Brunel, ${ }^{21}$ who derived and solved a (self-consistent) Fokker-Planck equation for the probability density of membrane potentials in a network of LIF neurons. The theory was developed by assuming a finite sparse connectivity, so that the thermodynamic limit is implicitly taken by letting the number of neurons diverge. As the resulting scenario - an asynchronous regime and two kinds of synchronous activity-does not fully match the one found 
in rate models, several numerical studies have been performed to investigate the role of ingredients such as the synaptic time scale or the network connectivity. ${ }^{11-14,22}$ The overall result is the evidence of some features which seem to conflict with the hypothesis of a widespread existence of a single "standard" asynchronous dynamics. For instance, Ostojic claims the existence of two different regimes that can be detected upon increasing the coupling strength. ${ }^{23}$ Even though this statement has been challenged by Engelken et al. ${ }^{24}$ who maintain that a single, standard, asynchronous regime does exist, the qualitative features of the spiking activity need to be better understood.

In this article, we revisit the activity of a balanced network of spiking neurons and propose a different interpretation of the regimes that have been observed in simulations of finite networks. Our approach is based on a thermodynamic limit, which better preserves the qualitative features observed in finite systems. All of our studies show that the network activity is not asynchronous but rather a manifestation of a collective irregular dynamics (CID), similar to what found in heterogeneous networks of globally coupled inhibitory neurons. ${ }^{25}$

Collective dynamics can be quantified by implementing the same indicators introduced to measure the degree of synchronization. With the help of an order parameter specifically designed to characterize neuronal synchrony in large ensembles of neurons, ${ }^{26}$ we find that CID is an ubiquituous phenomenon, which does not only persist for arbitrary coupling strength, but also in the absence of delay and refractoriness. Finally, we find that collective dynamics is not restricted to LIF neurons but extends at least to QIF neurons as well. All numerical calculations have been performed by implementing either an event-driven approach ${ }^{27,28}$ or Euler's algorithm.

We start considering an ensemble of $N$ supra-threshold LIF neurons composed of $b N$ excitatory and $(1-b) N$ inhibitory cells, as defined in Refs. 21 and 23. The membrane potential $V_{i}$ of the $i$ th neuron evolves according to the equation,

$$
\tau \dot{V}_{i}=R\left(I_{0}+I_{i}\right)-V_{i},
$$

where $\tau=20 \mathrm{~ms}$ is the membrane time constant, $R I_{0}=24 \mathrm{mV}$ is an external DC "current," and $R I_{i}$ is the synaptic current arising from the mutual coupling

$$
R I_{i}=\tau J \sum_{n} G_{i j(n)} \delta\left(t-t_{n}^{(j)}-\tau_{d}\right),
$$

where $J$ is the coupling strength. The synaptic connections among the neurons are random, with a constant in-degree $K$ for each neuron. The matrix elements assume the following values: $G_{i j}=1(-g)$, if the pre-synaptic neuron $j$ is excitatory (inhibitory), otherwise $G_{i j}=0$. If $V_{j}$ reaches the threshold $V_{t h}=20 \mathrm{mV}$ at time $t_{n}^{(j)}$, two events are triggered: (i) the membrane potential is reset to $V_{r}=10 \mathrm{mV}$ and $V_{j}$ is held fixed for a refractory period $\tau_{r}=0.5 \mathrm{~ms}$; (ii) a spike is emitted and received $\tau_{d}=0.55 \mathrm{~ms}$ later by the post-synaptic cells connected to neuron $j$. All the other parameters are initially set as in Ref. 23, namely $b=0.8, K=1000, g=5$, and $N=10000$.

We first compute the instantaneous probability density $P(v)$ of membrane potentials $V_{i} \in[v, v+d v]$ for $J=0.1 \mathrm{mV}$ and $0.5 \mathrm{mV}$. The asynchronous regime is by definition characterized by a constant firing rate ${ }^{29}$ (in the thermodynamic limit). This implies that the flux of neurons along the $v$-axis is independent of both potential and time, i.e., the corresponding probability density $P(v)$ is stationary. From Fig. 1, where three different snapshots of $P(v)$ are plotted, we notice instead strong fluctuations, which appear to grow with the coupling strength $J$.

Such large fluctuations are inconsistent with the stationarity of the asynchronous regime. In order to better understand their nature, it is necessary to take the thermodynamic limit. This can be done in various ways. In Ref. $21, N$ is let diverge to $\infty$, keeping all other parameters constant. This limit is not able to capture the fluctuations seen in Fig. 1, which indeed slowly vanish upon increasing $N$. In most of the literature on balanced states, ${ }^{10,12-14}$ first the limit $N \rightarrow \infty$ is taken, and then the average in-degree $K$ is let diverge under the assumption that the coupling strength $J$ is on the order of $\mathcal{O}(1 / \sqrt{K})$, i.e., one can rewrite explicitly $J=\mathcal{J} / \sqrt{K}$ and $R I_{0} \propto \sqrt{K}$. In this article, we propose to let $N$ and $K$ diverge simultaneously, assuming $K=c N$ (this corresponds to assuming a massive connectivity). A priori, there are two meaningful setups that can be considered: (W) weak external current, which corresponds to assume that $R I_{0}$ is independent of $N$ (and thereby $K$ ); (S) strong external current, i.e., $I_{0}=i_{0} \sqrt{N}$. In the (W) setup, the balance must be ensured a priori by imposing that excitatory and inhibitory fields nearly compensate each other. This is obtained by setting $g \equiv g_{0}+g_{1} / \sqrt{N}$ with $g_{0}=b /(1-b)$ so that the average difference between the excitation and inhibition is of the same order as statistical fluctuation. In the (S) setup, there is no need to tune $g$ because the external current $R I_{0}$ maintains the balance. In this article, we show that CID emerges in both setups.

We first report the results for increasing network sizes for the setup (W), starting from $N=10000$ and including 40000,160000 , and 640000 . We set $c=0.1$ and $g_{1}=100$,
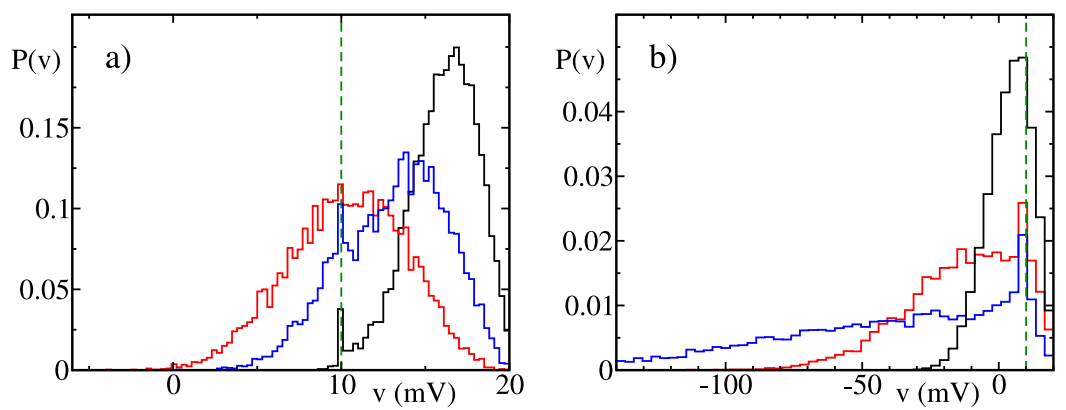

FIG. 1. Three different instantaneous probability distributions of the membrane potentials $P(v)$ for $N=10000$ and $J=0.1 \mathrm{mV}$ (a) and $J=0.5 \mathrm{mV}$ (b). The green dashed line refers to the reset potential $V_{r}$. 

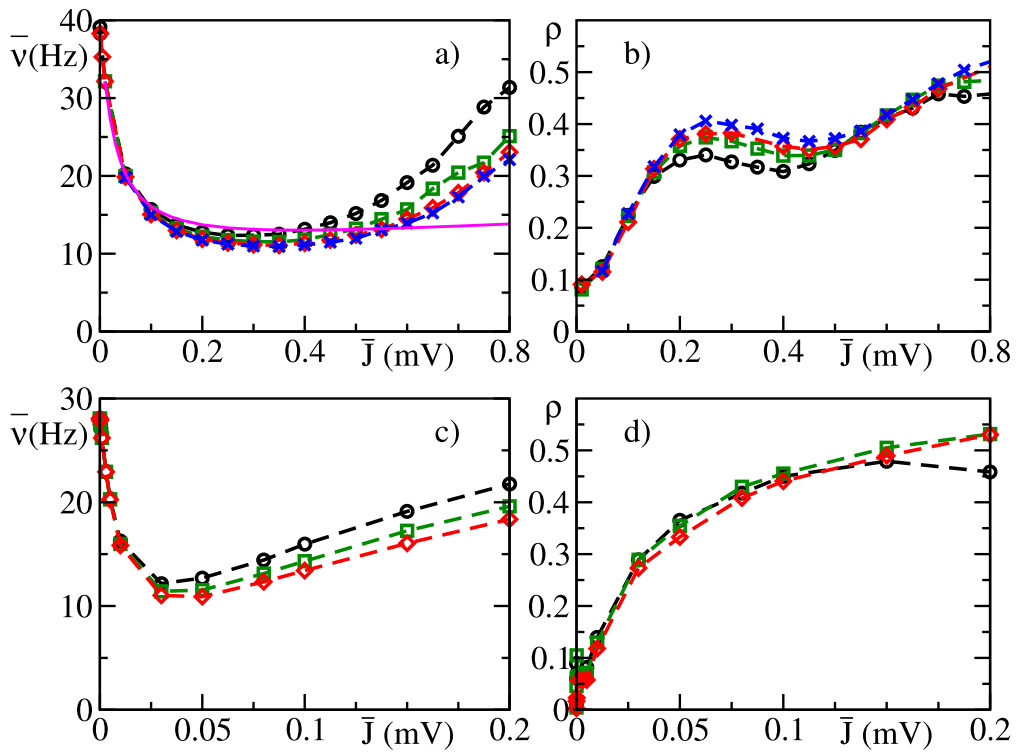

FIG. 2. Average firing rate $\bar{v}(\mathrm{a}, \mathrm{c})$ and order parameter $\rho(\mathrm{b}, \mathrm{d})$ versus the coupling strength $\bar{J}$ for different network sizes $N$ : black circles refer to $N=10000$, green squares to 40000 , red diamonds to 160000 , and blue crosses to 640000 [only in (a) and (b)]. The results refer to the (W) setup for $\operatorname{LIF}(a, b)$ and QIF (c,d). The indicators have been averaged over a time window of $40 \mathrm{~s}$ and over up to seven realizations of the network. The solid magenta line (a) is obtained by invoking the diffusion approximation for a noise driven LIF. $^{31}$ as the resulting model, for $N=10000$, is equivalent to that in Ref. 23. In Fig. 2(a) we plot the value of the average firing rate $\bar{v}$ versus $\bar{J}=\mathcal{J} / \sqrt{1000} .^{30}$ In order to damp the (small) sample-to-sample fluctuations, the results are averaged over seven, three, and two different realizations of the network for $N=10000,40000$, and 160000 , respectively. We observe a slow but clear convergence to an asymptotic curve in the entire range of coupling values. Finite-size corrections are negligible for $\bar{J}$ up to $0.1 \mathrm{mV}$, while for stronger coupling, the larger the network, the stronger is the tendency of the firing rate to decrease with the system size. Nevertheless, for $N \gtrsim 160000$, an asymptotic curve is attained, which exhibits a growth of $\bar{v}$ with $\bar{J}$ for sufficiently large coupling (compare with the solid full line, obtained by invoking the theoretical formula for a noise-driven $\mathrm{LIF}^{31}$ ). Another aspect that is maintained in the thermodynamic limit is a bursting activity, characterized by a coefficient of variation larger than $1,{ }^{32}$ for $\bar{J}>0.3 \mathrm{mV}$ (data not shown).

A typical order parameter that is used to quantify the strength of collective dynamics is based on the relative amplitude of the macroscopic fluctuations, ${ }^{26}$

$$
\rho^{2} \equiv \frac{\overline{\langle V\rangle^{2}}-\overline{\langle V\rangle}^{2}}{\left\langle\overline{V^{2}}-\bar{V}^{2}\right\rangle},
$$

where $\langle\cdot\rangle$ denotes an ensemble average, while the overbar is a time average. In practice, $\rho$ is the rescaled amplitude of the standard deviation of the average $\langle V\rangle$. When all neurons behave in exactly the same way (perfect synchronization), the numerator and the denominator are equal to one another and $\rho=1$. If instead they are independent, $\rho \approx 1 / \sqrt{N}$. From the results plotted in Fig. 2(b), we see that the order parameter $\rho$ is finite in the whole range of the considered coupling. Furthermore, it is substantially independent of $N$ for $\bar{J}<0.2 \mathrm{mV}$, while for larger $\bar{J}$, it exhibits a slower convergence to values $\simeq 0.4-0.5$. This clearly indicates that the thermodynamic phase is not a standard asynchronous regime but is rather characterized by a collective dynamics, also for very small coupling strengths.

The nature of the macroscopic dynamics can be appreciated from the spectrum $\mathcal{S}_{g}$ of the global activity $Y(t)$ (obtained by summing the signals emitted by all the neurons)-for different system sizes. In Fig. 3 we plot the rescaled spectrum $S_{g}=\mathcal{S}_{g} / N^{2}$ [panels (a) and (b) refer to $\bar{J}=0.2 \mathrm{mV}$ and $\bar{J}=0.8 \mathrm{mV}$, respectively]. The data collapse suggests that the dynamics remains irregular in the thermodynamic limit, i.e., that the fluctuations are not finite-size effects. In fact, an asynchronous regime would have been characterized by a spectral amplitude $\mathcal{S}_{g}$ of order $\mathcal{O}(N)$ rather than $\mathcal{O}\left(N^{2}\right)$. For both coupling strengths, the spectral density is mostly concentrated in
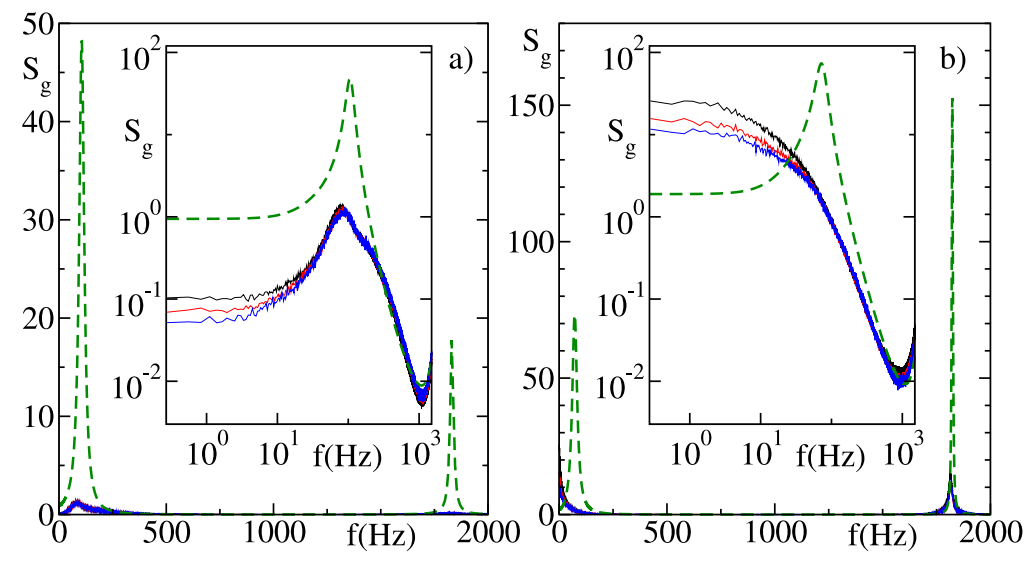

FIG. 3. Global spike-train spectra $S_{g}(f)$ versus the frequency for $\bar{J}=0.2 \mathrm{mV}$ (a) and $0.8 \mathrm{mV}$ (b) for LIF in (W) setup. The different lines refer to different system sizes, namely $N=10000$ (black), $N=40000$ (red), and $N=160000$ (blue). The dashed green lines show the theoretical results obtained by following Ref. 21. 
two frequency ranges: (i) around $f \approx 1800 \mathrm{~Hz}$, which corresponds to the inverse of the delay and (ii) at low frequencies in a range that approximately corresponds to the firing rate. A relative comparison confirms that the collective dynamics is stronger for larger coupling strengths.

Altogether, the broad-band structure of the spectrum suggests that the nature of the CID is stochastic-like even though the model is perfectly deterministic. The high-dimensional character of the neural activity is confirmed by a fractaldimension analysis. ${ }^{33,34}$ To our knowledge, we provide the first convincing evidence of collective irregular behaviour in a balanced state. The closest regime is reported in a preprint, ${ }^{35}$ which deals with a fully coupled rate model.

The persistence of collective dynamics in the thermodynamic limit in the (W) setup can be understood in the limit of small connectivity, by revisiting the theory developed by Brunel $^{21}$ in the context of highly-diluted networks, under the implicit assumption that the thermodynamic limit is taken by letting the number of neurons diverge. The central point is the evolution equation for the probability $P(v, t)$

$$
\tau \frac{\partial P}{\partial t}=\frac{\partial}{\partial v}[(v-\mu) P]+\frac{\sigma^{2}}{2} \frac{\partial^{2} P}{\partial v^{2}}+\sigma_{0} \sqrt{c \tau} \frac{\partial P}{\partial v} \zeta(t) .
$$

This stochastic Fokker-Planck equation was derived in Ref. 21 [see Eq. (32), here rewritten in our notations]; it is valid so long as the current $I$ can be represented as the sum of a deterministic contribution $\mu$ and a noise of amplitude $\sigma$. Self-consistent formulas for $\mu$ and $\sigma$ can be derived upon assuming an uncorrelated Poisson activity of the various neurons, obtaining $\mu(t)=R I_{0}+K J \tau(b-(1-b) g) v$ $\left(t-\tau_{d}\right)$ and $\sigma(t)=J \sqrt{\tau K\left(b+(1-b) g^{2}\right) v\left(t-\tau_{d}\right)}$ [see Eqs. (4) and (5) in Ref. 21], where $v(t)$ is the instantaneous firing rate at time $t^{36}$

The presence of the common noise in Eq. (4) is due to the sharing of a fraction of afferent neurons. In the context of Ref. 21 , the additive noise is a finite-size effect, since $c=K / N$ vanishes for $N \rightarrow \infty$, while in our

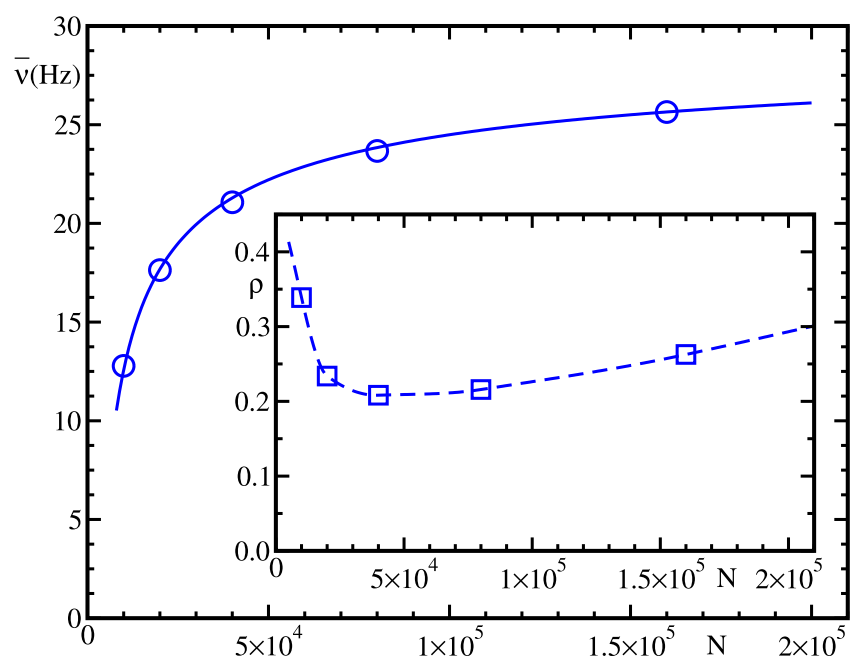

FIG. 4. Mean firing rate $\bar{v}$ as a function of the system size $N$ for $\bar{J}=0.2 \mathrm{mV}(\mathcal{J}=\bar{J} \sqrt{1000})$ for the LIF in (S) setup. The solid line, $\bar{v}=(30-1742.18 / \sqrt{N}) \mathrm{Hz}$, shows the convergence to the expected asymptotic value $\bar{v}=30 \mathrm{~Hz}$. The inset shows the dependence of the synchronisation measure $\rho$ on $N$ (the dashed line is a guide for the eyes). setup it remains finite. More precisely, $\zeta(t)$ is a white noise term- $\langle\zeta(t) \zeta(t+T)\rangle=\delta(T)$-while $\sigma_{0}$ is the value of $\sigma$ corresponding to the constant firing rate $\nu_{0}$ obtained within the diffusion approximation. ${ }^{31}$ By inserting our scaling assumptions for $K, J$, and $g$, we find that in the thermodynamic limit $\mu(t)=R I_{0}-\mathcal{J} \tau \sqrt{c} g_{1}(1-b) \nu\left(t-\tau_{d}\right)$ and $\sigma(t)=\mathcal{J} \sqrt{b \tau \nu\left(t-\tau_{d}\right) /(1-b)}$, i.e., both parameters remain finite when the limit $N \rightarrow \infty$ is taken. As a result, it makes sense to use the solution of Eq. (4) as a reference for the results of our numerical simulations. An analytic expression for the rescaled power spectrum can be found in Ref. 21 (see the expression reported at the end of page 204). The resulting shape for our parameter values is reported in Fig. 3 [see the green dashed line in panels (a) and (b)]. A qualitative agreement is observed for both $\bar{J}=0.2 \mathrm{mV}$ and $\bar{J}=0.8 \mathrm{mV}$, starting from the presence of a peak in correspondence of the inverse delay. The similarity between the low-frequency peaks is less pronounced for the higher coupling strength, showing that the true dynamics is definitely less regular than theoretically predicted by the noisy Fokker-Planck equation. The lack of a quantitative agreement is not a surprise, given the perturbative character of the noisy term and the assumption of a Poisson statistics that is not generally valid.

A closer agreement would be obtained if we could relax some of such approximations. A promising approach is the self-consistent method developed by Lindner and coauthors, ${ }^{37,38}$ which provides a more accurate description of the spiking activity of LIF neurons. Unfortunately, for our setup above $\bar{J}=0.05 \mathrm{mV}$, the method does not converge, ${ }^{39}$ thus leaving open the question whether it is a technical or conceptual matter.

We now discuss the strong-current setup (S), with $R I_{0}=0.24 \sqrt{N} \mathrm{mV}$ (i.e., $R i_{0}=0.24 \mathrm{mV}$ ) and $g=5$. In this case, the balance is attained (at leading order in $N$ ) by imposing the condition $I_{i}+I_{0}=0$. Under the assumption of a constant firing rate, this implies $\bar{v}=R i_{0} /(\sqrt{c} \tau \mathcal{J}((1-b) g-b))$. The results of simulations for $\bar{J}=0.2 \mathrm{mV}$ and different values of $N$ are reported in Fig. 4, where one can see that the firing rate converges toward the expected asymptotic value $\bar{v}=30 \mathrm{~Hz}$, with a $1 / \sqrt{N}$ rate (see the solid line). More important is that the order parameter $\rho$ remains finite for increasing $N$ (see the inset). The presence of strong finite-size corrections prevents us from determining its asymptotic value; it is, however, clear that $\rho$ does not vanish, indicating that a collective dynamics emerges also in the presence of strong external currents.

In order to establish the generality of CID in balanced, massively coupled networks, we have analysed another model, the QIF, which represents the canonical model for class I excitability. ${ }^{16,40,41}$ Its evolution equation reads as

$$
\tau \dot{\theta}_{i}=\left(1+\cos \theta_{i}\right)+\left(1-\cos \theta_{i}\right)\left(\theta_{0}+\alpha R I_{i}\right),
$$

where $\theta_{i}$ is an adimensional phase-like variable and $\theta_{t h}=\pi$ and $\theta_{r}=-\pi$ are the threshold and reset value, respectively. Moreover, $\theta_{0}=0.2, \alpha=1 \mathrm{mV}^{-1}$, while $R I_{i}$ is still defined as in Eq. (2), and all the other parameters are as for the LIF. As shown in Figs. 2(c) and 2(d), where the firing rate $\bar{v}$ and the order parameter $\rho$ are reported for different coupling strengths, there is again a clear evidence of synchronization. 
The broadband structure of the corresponding spectra of the neural activity (data not shown) indicates that the collective dynamics is stochastic-like.

Finally, we made several other tests, eliminating refractoriness, setting the delay equal to zero and adding noise to the external current (as in the original Brunel paper ${ }^{21}$ ). In all these cases, $\rho$ remains finite and exhibits an irregular behavior. ${ }^{33}$

Altogether, we have found that CID emerges in all massively coupled networks that we have explored. This comes as a surprise: in other models of massively coupled neuronal systems, the microscopic chaotic dynamics, which may emerge in finite systems, disappears in the thermodynamic limit, ${ }^{42,43}$ while here it does not only survive but contributes to sustain a macroscopic stochastic-like evolution. This point definitely needs to be better clarified.

The evidence that CID survives in the vanishing coupling limit could represent the starting point for future progress. In fact, for $J=0$ any distribution $P(v)$ is a marginally stable solution and can in principle be (de)stabilized by an arbitrarily small coupling. Such a singular behavior was successfully handled to explain the onset of partial synchrony, ${ }^{4}$ by mapping the ensemble of LIF neurons onto the much simpler Kuramoto-Daido equation. ${ }^{44,45}$ Can one hope to make a similar analysis in the context of the balanced regime?

The authors acknowledge N. Brunel, F. Farkhooi, G. Mato, M. Timme, and M. di Volo for enlightening discussions. This work has been partially supported by the

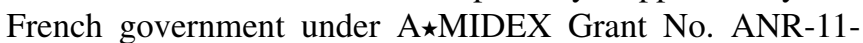
IDEX-0001-02 (A.T.) and mainly realized at the Max Planck Institute for the Physics of Complex Systems (Dresden, Germany) within the activity of the Advanced Study Group 2016/17 "From Microscopic to Collective Dynamics in Neural Circuits."

${ }^{1}$ A. T. Winfree, The Geometry of Biological Time, 2nd ed., Interdisciplinary Applied Mathematics Vol. 12 (Springer-Verlag, New York, 2001).

${ }^{2}$ J. A. Acebrón, L. L. Bonilla, C. J. Pérez Vicente, F. Ritort, and R. Spigler, Rev. Mod. Phys. 77, 137 (2005).

${ }^{3}$ M. J. Panaggio and D. M. Abrams, Nonlinearity 28, R67 (2015).

${ }^{4}$ C. van Vreeswijk, Phys. Rev. E 54, 5522 (1996).

${ }^{5}$ D. Golomb, D. Hansel, B. Shraiman, and H. Sompolinsky, Phys. Rev. A 45, 3516 (1992).

${ }^{6}$ Dynamics and Thermodynamics of Systems with Long Range Interactions, Lecture Notes in Physics Vol. 602, edited by T. Dauxois, S. Ruffo, E. Arimondo, and M. Wilkens (Springer-Verlag, Berlin, Heidelberg, 2002).
${ }^{7}$ M. Mézard, G. Parisi, and M. Virasoro, Spin Glass Theory and Beyond: An Introduction to the Replica Method and Its Applications (World Scientific Publishing Co. Inc., 1987), Vol. 9.

${ }^{8}$ A. Crisanti and H. Sompolinsky, Phys. Rev. A 37, 4865 (1988).

${ }^{9}$ H. Sompolinsky, A. Crisanti, and H. J. Sommers, Phys. Rev. Lett. 61, 259 (1988).

${ }^{10}$ C. van Vreeswijk and H. Sompolinsky, Science 274, 1724 (1996).

${ }^{11}$ A. Renart, J. de la Rocha, P. Bartho, L. Hollender, N. Parga, A. Reyes, and K. D. Harris, Science 327, 587 (2010).

${ }^{12}$ A. Litwin-Kumar and B. Doiron, Nat. Neurosci. 15, 1498 (2012).

${ }^{13}$ J. Kadmon and H. Sompolinsky, Phys. Rev. X 5, 041030 (2015).

${ }^{14}$ O. Harish and D. Hansel, PLoS Comput. Biol. 11, e1004266 (2015).

${ }^{15}$ F. Farkhooi and W. Stannat, Phys. Rev. Lett. 119, 208301 (2017).

${ }^{16}$ B. S. Gutkin and G. B. Ermentrout, Neural Comput. 10, 1047 (1998).

${ }^{17}$ A. Rauch, G. La Camera, H.-R. Lüscher, W. Senn, and S. Fusi, J. Neurophysiol. 90, 1598 (2003).

${ }^{18}$ R. Jolivet, T. J. Lewis, and W. Gerstner, J. Neurophysiol. 92, 959 (2004).

${ }^{19}$ R. Jolivet, A. Rauch, H.-R. Lüscher, and W. Gerstner, J. Comput. Neurosci. 21, 35 (2006).

${ }^{20}$ E. Shlizerman and P. Holmes, Neural Comput. 24, 2078 (2012).

${ }^{21}$ N. Brunel, J. Comput. Neurosci. 8, 183 (2000).

${ }^{22}$ R. Rosenbaum and B. Doiron, Phys. Rev. X 4, 021039 (2014).

${ }^{23}$ S. Ostojic, Nat. Neurosci. 17, 594 (2014).

${ }^{24}$ R. Engelken, F. Farkhooi, D. Hansel, C. van Vreeswijk, and F. Wolf, F1000Research 5, 2043 (2016).

${ }^{25}$ S. Luccioli and A. Politi, Phys. Rev. Lett. 105, 158104 (2010).

${ }^{26}$ D. Golomb, Scholarpedia 2, 1347 (2007).

${ }^{27}$ R. Zillmer, R. Livi, A. Politi, and A. Torcini, Phys. Rev. E 74, 036203 (2006).

${ }^{28}$ M. Rudolph and A. Destexhe, Neurocomputing 70, 1966 (2007).

${ }^{29}$ W. Gerstner, W. M. Kistler, R. Naud, and L. Paninski, Neuronal Dynamics: From Single Neurons to Networks and Models of Cognition (Cambridge University Press, Cambridge, 2014).

${ }^{30}$ This variable has a one-to-one correspondence with $J$ in Ref. 23.

${ }^{31}$ R. Capocelli and L. Ricciardi, Kybernetik 8, 214 (1971).

${ }^{32}$ E. M. Izhikevich, Dynamical Systems in Neuroscience (MIT Press, 2007).

${ }^{33}$ A. Politi, E. Ullner, A. Torcini, "Collective irregular dynamics in balanced networks of leaky integrate-and-fire neurons," Eur. Phys. J. Spec. Top. (in press); preprint arXiv:1808.03359.

${ }^{34}$ A. Torcini, A. Politi, G. Puccioni, and G. D’Alessandro, Physica D 53, 85 (1991).

${ }^{35}$ T. Hayakawa and T. Fukai, preprint arXiv:1711.09621 (2017).

${ }^{36}$ The above equation must be complemented by suitable b.c. to take into account refractoriness and the reset.

${ }^{37}$ B. Dummer, S. Wieland, and B. Lindner, Front. Comput. Neurosci. 8, 104 (2014).

${ }^{38}$ S. Wieland, D. Bernardi, T. Schwalger, and B. Lindner, Phys. Rev. E 92, 040901 (2015).

${ }^{39}$ Problems of stability of the method for large inhibition (namely, $g=5$ ) have been previously reported also by the authors in Ref. 37 .

${ }^{40}$ B. Ermentrout, Scholarpedia 3, 1398 (2008).

${ }^{41}$ M. Monteforte and F. Wolf, Phys. Rev. Lett. 105, 268104 (2010).

${ }^{42}$ S. Olmi, R. Livi, A. Politi, and A. Torcini, Phys. Rev. E 81, 046119 (2010).

${ }^{43}$ L. Tattini, S. Olmi, and A. Torcini, Chaos 22, 023133 (2012).

${ }^{44}$ H. Daido, Physica D 69, 394 (1993).

${ }^{45}$ A. Politi and M. Rosenblum, Phys. Rev. E 91, 042916 (2015). 